\title{
Electromechanical Design of Self-Similar Inspired Surface Electrodes for Human-Machine Interaction
}

\author{
YongAn Huang $\mathbb{D}^{1,2}$ Wentao Dong, ${ }^{1,3}$ Chen $\mathrm{Zhu}^{1,2}$ and Lin Xiao ${ }^{1,2}$ \\ ${ }^{1}$ State Key Laboratory of Digital Manufacturing Equipment and Technology, Huazhong University of Science and Technology, \\ Wuhan 430074, China \\ ${ }^{2}$ Flexible Electronics Research Center, Huazhong University of Science and Technology, Wuhan 430074, China \\ ${ }^{3}$ School of Electrical and Automation Engineering, East China Jiaotong University, Nanchang 330013, China
}

Correspondence should be addressed to YongAn Huang; yahuang@hust.edu.cn

Received 20 March 2018; Revised 13 May 2018; Accepted 30 May 2018; Published 13 August 2018

Academic Editor: Zhaojie Ju

Copyright (C) 2018 YongAn Huang et al. This is an open access article distributed under the Creative Commons Attribution License, which permits unrestricted use, distribution, and reproduction in any medium, provided the original work is properly cited.

\begin{abstract}
Stable acquisition of electromyography (EMG)/electrocardiograph (ECG) signal is critical and challenging in dynamic humanmachine interaction. Here, self-similar inspired configuration is presented to design surface electrodes with high mechanical adaptability (stretchability and conformability with skin) and electrical sensitivity/stability which are usually a pair of paradoxes. Mechanical and electrical coupling optimization strategies are proposed to optimize the surface electrodes with the 2nd-order self-similar serpentine configuration. It is devoted the relationship between the geometric shape parameters (height-space ratio $\eta$, scale factor $\beta$, and line width $w$ ), the areal coverage $\alpha$, and mechanical adaptability, based on which an open network-shaped electrode is designed to stably collect high signal-to-noise ratio signals. The theoretical and experimental results show that the electrodes can be stretched $>30 \%$ and conform with skin wrinkle. The interfacial strength of electrode and skin is measured by homemade peeling test experiment platform. The surface electrodes with different line widths are used to record ECG signals for validating the electrical stability. Conformability reduces background noises and motion artifacts which provides stable recording of ECG/EMG signals. Further, the thin, stretchable electrodes are mounted on the human epidermis for continuous, stable biopotential signal records which suggests the way to highperformance electrodes in human-machine interaction.
\end{abstract}

\section{Introduction}

Surface electrodes that can acquire electrophysiological (EP) signals have many applications ranging from diagnosing neuromuscular disorders $[1,2]$, artificial muscle $[3,4]$, to stimulation of hand rehabilitation [5]. The conventional electrodes are fixed on the body surface by caps, belts, or tapes [6], which lead to inconvenient life of the people as their relatively thick and rigid nature, limited options in mounting locations, and discomfort in long-term usage on the soft, curved, and dynamic human body [7]. The ideal surface electrode is desired to have excellent mechanical adaptability (stretchability $>30 \%$, and conformability with skin wrinkles) and keep electrical stability in recording EP signals during body motion.
Material selection and structural design strategies for stretchable electrodes were proposed to improve the mechanical flexibility and the strength of EP signals [8-13]. An electrode with adhesive patch of modulus-tunable composite was proposed to health monitoring [14-16]. Epidermal strain sensor with improved PEIE-polydimethylsiloxane (S3PDMS) elastomer substrate showed excellent compatibility with human skin $[17,18]$. Optimized structural designs for large deformation were validated by experimental and theoretical results and applied in flexible/stretchable electronics [19-21]. The "bridge-island" structure was proposed to enhance both the stretchability of bridge and the area coverage of island for the stretchable electronics [22, 23]. Optimal geometric parameters of filamentary serpentine structure of surface electrode, such as the thickness of substrate, 


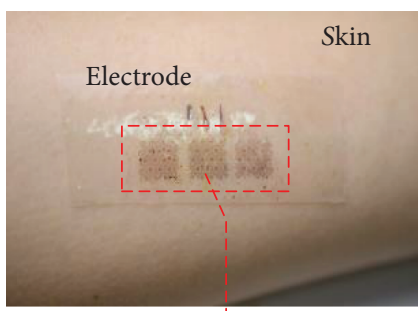

(a)

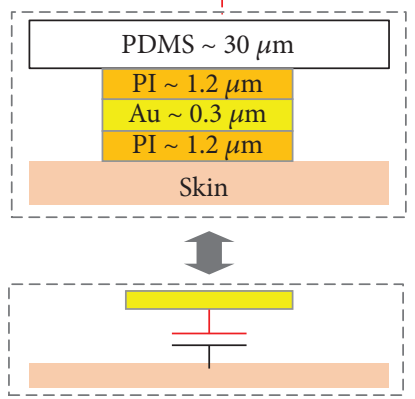

(c)

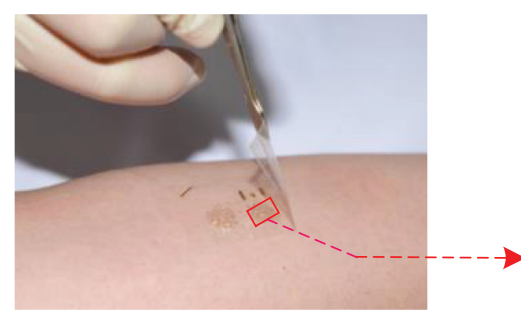

(b)

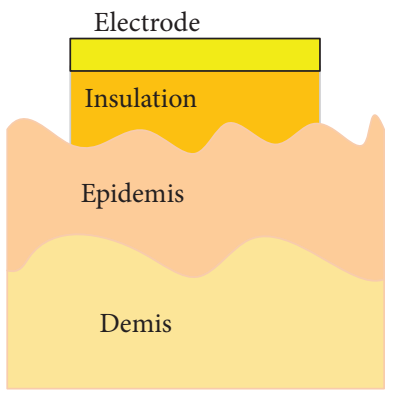

(d)

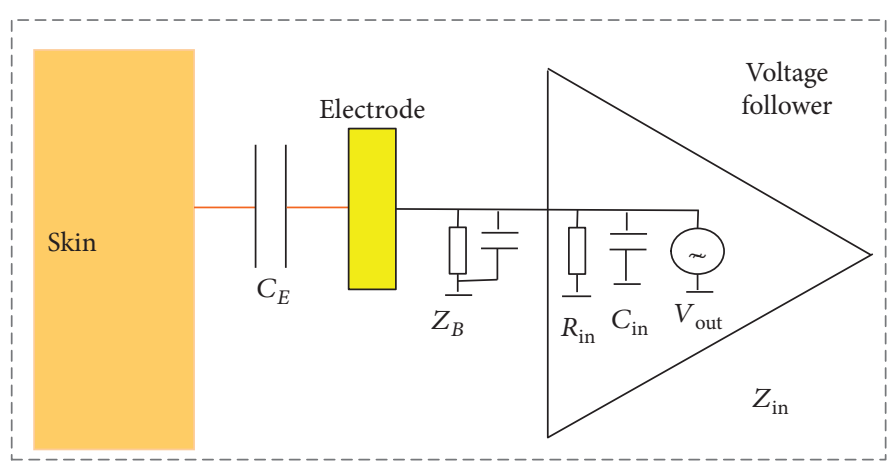

(e)

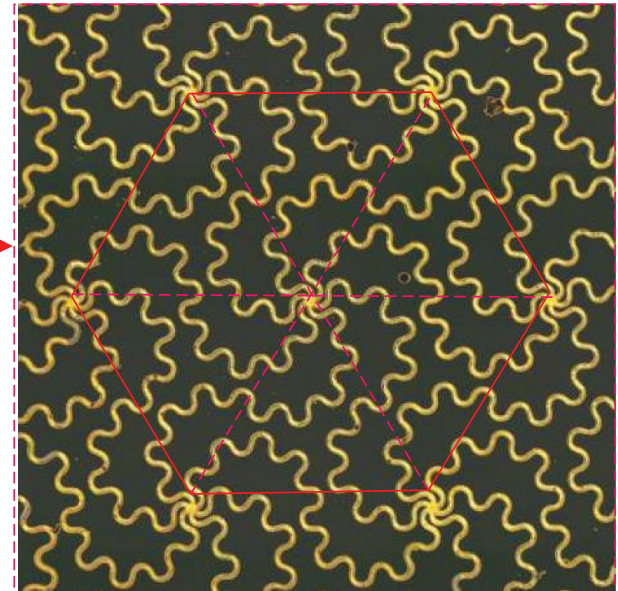

(f)

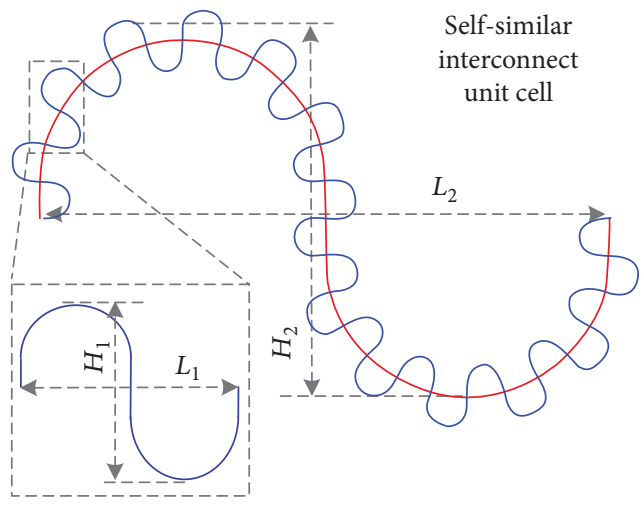

(g)

FIGURE 1: Self-similar inspired surface electrode: (a) the electrode laminated on the skin surface conformally; (b) the electrode delaminated from the skin surface; (c) and (d) schematic graph of the skin-electrode interface via capacitive coupling; (e) data acquisition circuit for the electrode with a voltage follower; (f) network-shaped electrode with the second-order serpentine structure; and (g) geometry parameters of the microstructure with the 1st- and 2nd-order serpentine interconnect.

interelectrode distance, line width, and radius of the microstructure, were considered to improve the electrical performance $[8,24]$. Self-similar serpentine interconnects were adopted in network-shaped surface electrode of EP sensor, to satisfy large deformation as the skin motion [25-27]. It was mainly attributed to the tremendous reduction of stiffness and increasing of length of the 2ndorder structure compared to the 1st-order one [21, 28, 29]. The structural optimization of surface electrode was merely focused on the stretchability and conformability, respectively $[8,25]$. However, the collaborative optimization of the mechanical and electrical properties has not been reported for stretchable surface electrodes.

This paper proposes a design strategy of the self-similar inspired biopotential electrode, which satisfies mechanical adaptability and electrical performance. The optimization model is to simultaneously improve the stretchability up to $>30 \%$, the conformability with skin wrinkles, the output signal gain, and the signal-noise-ratio (SNR) of surface electrodes used in electromyography (EMG)/electrocardiograph (ECG) signal recordings. The theoretical analysis and experimental results indicate that the self-similar design effectively improves the stretchability, conformability, areal coverage, and electrical performance of the surface electrode. Further, the signal stability is validated through the biological signal recordings in the case of walking and running. The surface electrodes which conformally contact with the skin surface can reduce the motion artifacts for stable biological signal recordings. It firstly provides a structural design method for stretchable electrodes with high deformability, conformability, areal coverage, and recording stability of EP signals.

\section{Self-Similar Inspired Design}

Figure 1(a) depicts the optical image of the surface capacitive electrode conformally mounted on the human skin. The surface capacitive electrode measures EP signals by embedding 
PI polymer dielectric layer between the electrode and skin. The electrode is easily delaminated from the skin with tweezers (Figure 1(b)), which provides an effective way to recycle the electrodes. Figure 1(c) demonstrates the electrode with multilayer structure of PDMS $(\sim 30 \mu \mathrm{m})$, PI $(\sim 1.2 \mu \mathrm{m})$, $\mathrm{Au}(\sim 0.3 \mu \mathrm{m})$, and PI $(\sim 1.2 \mu \mathrm{m})$. The capacitance at the skin-electrode interface is represented as $C_{E}=\varepsilon_{\mathrm{r}} \varepsilon_{0} S / d$, where $\varepsilon_{\mathrm{r}}$ represents the relative dielectric constant, $\varepsilon_{0}$ is vacuum dielectric constant, $S$ denotes the area of the electrode, and $d$ denotes the distance between the skin and electrode. The Au layer locates at the neutral plane of the electrode for reducing the strain of Au layer. Figure 1(d) shows that the stretchable surface electrode follows the wrinkles of the skin surface.

Figure 1(e) depicts the equivalent circuit for data acquisition of the surface electrode with a voltage follower. Higher signal gain is realized by using higher input impedance, and low-input capacitance is adopted to improve the signal quality. Figure 1(f) depicts the layout of wavy network-shaped with the 2 nd-order self-similar serpentine electrode consisting of horseshoe building blocks. The node connection of the unit cells in the network-shaped electrode forms triangular lattices. Figure 1(g) illustrates the geometry parameters of microinterconnect for the 1st- and 2nd-order serpentine structure. The heights of the 1st-order and the 2nd-order serpentine interconnects are $H_{1}$ and $H_{2}$, respectively. And the line width and the thickness are $w$ and $t$, respectively. As the shape of the 1st- and 2nd-order serpentine geometries is almost the same, that is, height-space ratio $\eta=2 H_{1} / L_{1}=2 H_{2} / L_{2}$. The scale factor $\beta=L_{2} / L_{1}$ represents the ratio of the 2 nd-order serpentine space to the 1storder space. The geometry of the 2nd-order serpentine structure is characterized by base length $\left(L_{1}, w, t\right)$ and two nondimensional parameters $(\eta, \beta)$, where $t$ is usually determined by the magnetron sputtering process. Both the mechanical adaptability and electrical performance depend on the geometry parameters of the electrodes but usually show competing relationship.

\section{Electromechanical Optimization Model}

The surface capacitive electrodes are designed based on self-similar serpentine geometry. The electromechanical optimization model is built to improve the electrical performance for satisfying excellent output gain, deformation, and conformability with the skin. As the principle of capacitive electrode shown in Figure 1(e), the transfer function model for the output gain of the electrode is represented as $G(s)=V_{\text {out }}(\mathrm{s}) / V_{\text {in }}(\mathrm{s})=\left(Z_{\text {in }} / / Z_{B}\right) /\left(Z_{E}+Z_{\text {in }} / / Z_{B}\right) \approx$ $\left(R_{B}+s C_{E} R_{B} R_{E}\right) /\left(\left(R_{B}+R_{E}\right)+s\left(C_{B}+C_{E}\right) R_{B} R_{E}\right)$, where $R_{\text {in }}$ and $C_{\text {in }}$ are the resistance and capacitance of the voltage follower. As $R_{E}>>R_{B}$, the transfer function model can be represented as [30]

$$
G(\mathrm{~s})=\frac{s C_{E} R_{B}}{1+s\left(C_{B}+C_{E}\right) R_{B}},
$$

where $C_{E}=\varepsilon S_{\text {effective }} / h_{\mathrm{PI}}=\varepsilon \alpha(\eta, \beta, w) S_{\text {electrode }} / h_{\mathrm{PI}}$ is the capacitance of the electrode-PI-skin capacitor, $R_{B}$ is a bias resistance between the ground and the operational amplifier. The output signal gain $G$ depends on the capacitance $C_{\mathrm{E}}$ with given voltage follower. The 2-norm of the transfer function (1) is represented as

$$
\|G\|=\frac{C_{E} R_{B}}{\sqrt{1+\left(C_{B}+C_{E}\right)^{2} R_{B}^{2}}} .
$$

Interfacial mechanics model is built to analyze the contact behavior at the electrode-skin interface, and the critical criteria to guarantee the electrode conformal contact with the skin is provided as $[19,31]$

$$
\alpha_{\text {electrode }}(\eta, \beta, w)<1-\frac{1}{\left(\left(16 / \bar{E}_{\text {skin }}\right)+\left(\lambda_{\text {rough }}^{3} / \overline{E_{\text {electrode }}} \pi^{3}\right)\right)} \frac{2 h_{\text {rough }}^{2} \pi}{\gamma \lambda_{\text {rough }}},
$$

where $w$ is the line width of the surface electrode, and $h_{\text {rough }}$ and $\lambda_{\text {rough }}$ denote the characteristic amplitude and wavelength of the skin roughness, respectively. Here, the adhesion force $\gamma$ at skin-electrode interface is measured by the homemade peeling platform, and the experiment process is discussed in (Appendix B).

The stretchability $\varepsilon_{\text {stretchability }}$ of the self-similar inspired microinterconnect unit depends on the geometry parameters $\eta, \beta, w / L_{1}$, and $t / L_{1}$, which is expressed with the scale law formula [32-34]:

$$
\varepsilon_{\text {stretchablity }}=F\left(\eta, \beta, \frac{w}{L_{1}}, \frac{t}{L_{1}}, \widehat{\varepsilon}\right) .
$$

The mapping relationship between the stretchability of the network-shaped electrode $\varepsilon_{\text {electrode-stretchability }}$ and the self-similar inspired microinterconnect unit $\varepsilon_{\text {stretchability }}$ can be written as [35]

$$
\varepsilon_{\text {electrode-stretchability }}=\varepsilon_{\text {stretchability }} \times\left(1-\sqrt{\alpha_{\text {electrode }}}\right) .
$$

The electromechanical optimization model (2), (3), (4), and (5) that integrates the coupling mechanical and electrical properties of the electrode is built to improve the mechanical adaptability and electrical performance. It is observed that the model is related to the geometry structure parameters $\eta, \beta$, and $w$. Design strategies are provided to realize with high filling factors and stretchability of the surface electrode. It is difficult to solve the complicated optimization model with multiple objectives and constraints directly. Multistep method is proposed to solve the optimization model (2), (3), (4), and (5), where the flow chart is shown in Figure 2, mainly including three steps: (1) explaining the competing relationship between areal coverage and stretchability of the microinterconnect unit for providing a relatively larger feasible range; (2) the effects of the layout of surface electrode on the output gain for determining the area of the electrode; and (3) devoting the relationship between stretchability and conformability to providing the 


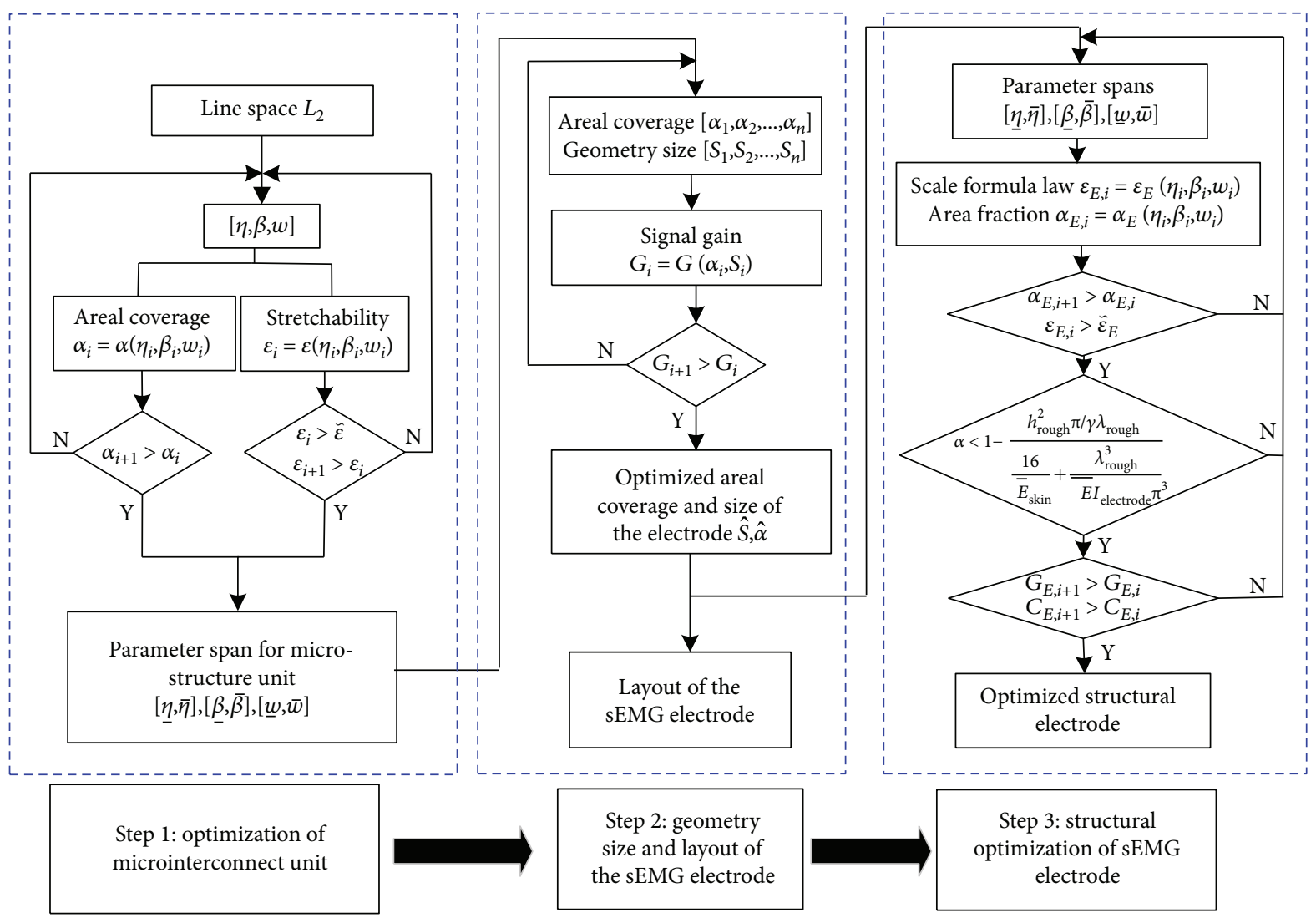

FIgURE 2: Solving process chart for the optimized structural design of surface electrode.

higher electrical performance and determining the feasible geometry parameter range of the surface electrode.

\subsection{Competing Relationship between Areal Coverage and} Stretchability. There are two important factors, areal coverage and stretchability, used to evaluate the electrical and mechanical performances of the stretchable surface electrode. Step 1 in Figure 2 depicts the solving process for the optimization of the microinterconnect unit. Given the space $L_{1}$ of the microstructure unit, the geometry parameters $\eta$, $\beta$, and $w$ are optimized to balance the areal coverage and the stretchability.

Figure 3(a) illustrates the effect of line width $w$ on the stretchability and the areal coverage of microinterconnect unit. The scatter points are from FEM simulation, and the red curve is from the theoretical analysis [20]. Given $\eta=1.25$ and $\beta=8$, the areal coverage linearly increases with the line width $w$, and the stretchability monotonously decreases with the line width $w$. For areal coverage and $\varepsilon_{\text {stretchability }}>35 \%$, the line width $w$ ranges from $30 \mu \mathrm{m}$ to $72 \mu \mathrm{m}$. Figure 3(b) depicts that the stretchability and the areal coverage of microinterconnect unit vary with the height-space ratio $\eta$. The scatter points are from FEM simulation, and the red curve is from the theoretical analysis. The areal coverage decreases with $\eta$ by theoretical analyses and experiments, and the stretchability of microstructure unit monotonically increases with $\eta$. Given $\beta=8$ and $w=50 \mu \mathrm{m}$, for $\varepsilon_{\text {stretchability }}>35 \%$, height-space ratio $\eta$ is larger than 1.2. Figure 3(c) depicts the relationship between the stretchability and areal coverage of microinterconnect unit with scale ratio $\beta$. The stretchability decreases with increasing $\beta$, and it approaches to $28 \%$ (the stretchability of the 1st-order serpentine structure). Given $\eta=1.25$ and $w=50 \mu \mathrm{m}$, for $\varepsilon_{\text {stretchability }}>35 \%, \beta$ is smaller than 20 .

\subsection{Layout of Surface Electrodes. Step 2 in Figure 2 illus-} trates how to determine the layout of the self-similar inspired surface electrode based on the triangular lattices. The voltage follower with the input impendence $R_{\mathrm{B}}=1$ $\mathrm{G} \Omega$ and $C_{\mathrm{in}}=100 \mathrm{pF}$ is shown in Figure $1(\mathrm{e})$. The output gain of the surface electrode depends on the capacitance $C_{\mathrm{E}}$ at skin-electrode interface, as shown in (2). Figure 4(a) reflects the effect of area (the area of metal film) on the electrical performance (normalized gain) of the surface electrode, where the areal coverage $\alpha=0.1,0.2$, $0.3,0.4$, and 0.5 , respectively. The output gain increases with the effective area of the electrode. When the areal coverage $\alpha=0.1$, it is difficult to reach the objective of the output gain $G(>0.95)$. Figure $4(\mathrm{~b})$ shows that the output gain increases with the increasing area with different $\eta$, and it reaches capacity with $S>25 \mathrm{~mm}^{2}$, which provides a guideline to determine the electrode area. As the surface electrode with the second-order serpentine interconnect forms into the triangular lattice structure, 


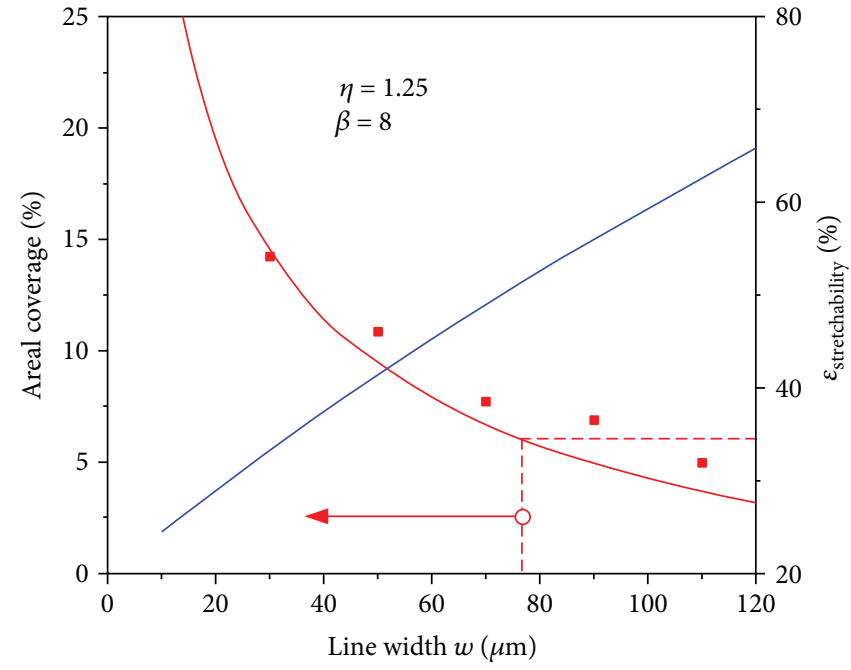

(a)

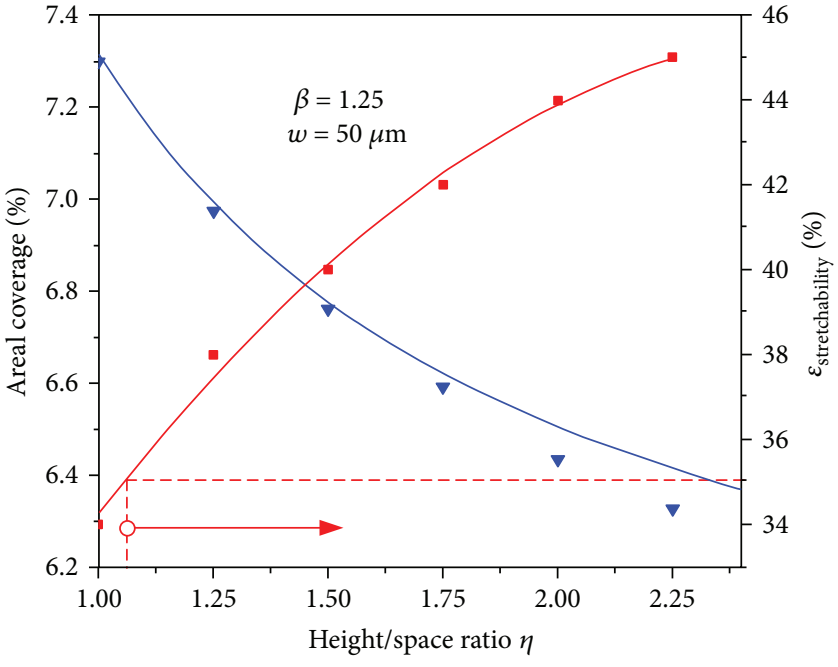

(b)

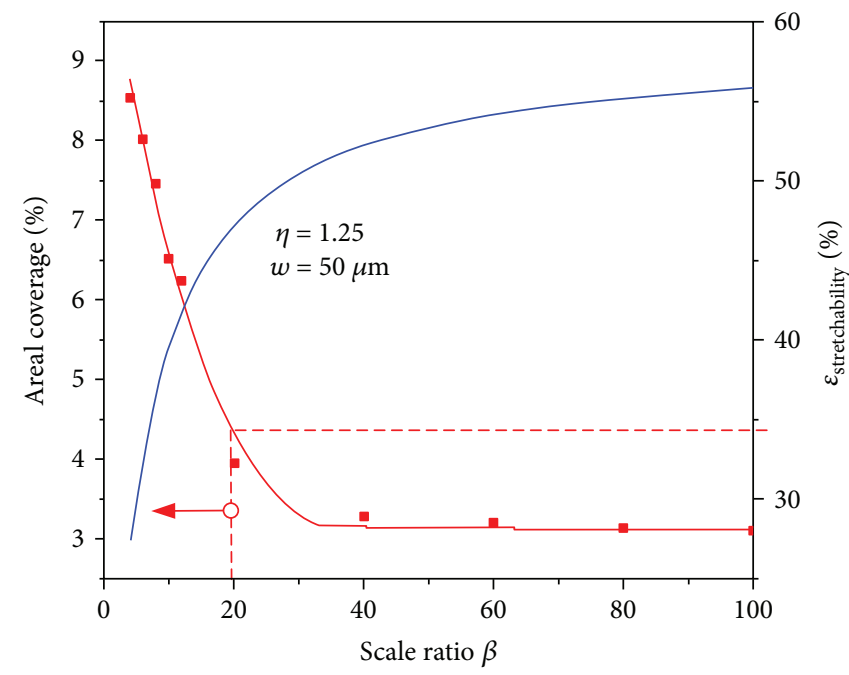

(c)

FIGURE 3: The effects of geometry parameters of the microinterconnect structure on the areal coverage and stretchability. (a) Line width $w$; (b) height-space ratio $\eta$; and (c) scale ratio $\beta$.

number of nodes are also computed for the electrode with the given area of the surface electrode, and so is the layout of the surface electrode.

3.3. Competing Relationship between Stretchability and Conformability. The feasible range of geometry parameters of microinterconnect under the constraints of stretchability and areal coverage given in Figure 3 provides a selection range for structural optimization of the surface electrode. The effect of line width $w$ on the stretchability and conformability is presented in Figure 5(a). It can be observed from (3) the surface electrode conformally contacts with the skin when the width $w$ is smaller than $72 \mu \mathrm{m}$. When it is smaller than $65 \mu \mathrm{m}$, the stretchability is larger than $30 \%$ by the scale formula (4). For the electrode with high filling factor to detect biological signals, the line width $w$ should be larger than $30 \mu \mathrm{m}$. So the feasible range of line width $w$ is $(30 \mu \mathrm{m}, 65 \mu \mathrm{m})$.
Figure 5(b) depicts the effect of height-space ratio $\eta$ on the stretchability and conformability. The stretchability and the total energy (sum of the bending and elastic energy of the electrode, skin energy, and the adhesion energy) at the electrode-skin interface increase with $\eta$. The surface electrode can conformally contact with the skin surface at the case of $\eta<2.25$, and the stretchability is larger than $30 \%$ by (4) when $\eta>1.2$. For the conformal contact and stretchability, the feasible range of $\eta$ is $(1.2,2.25)$.

Figure 5(c) is a depiction on the effect of scale factor $\beta$ on the stretchability and conformability. When it is smaller than 16 , the stretchability is larger than $30 \%$ by (4). When $\beta$ is smaller than 10 , the electrode conformally contacts with the skin. As the space of the 2nd-order structure is four times larger than the space of the first order, $\beta$ is larger than 4 . For the conformal contact behavior and stretchability, the feasible range of geometry parameter $\beta$ is $(4,10)$. 


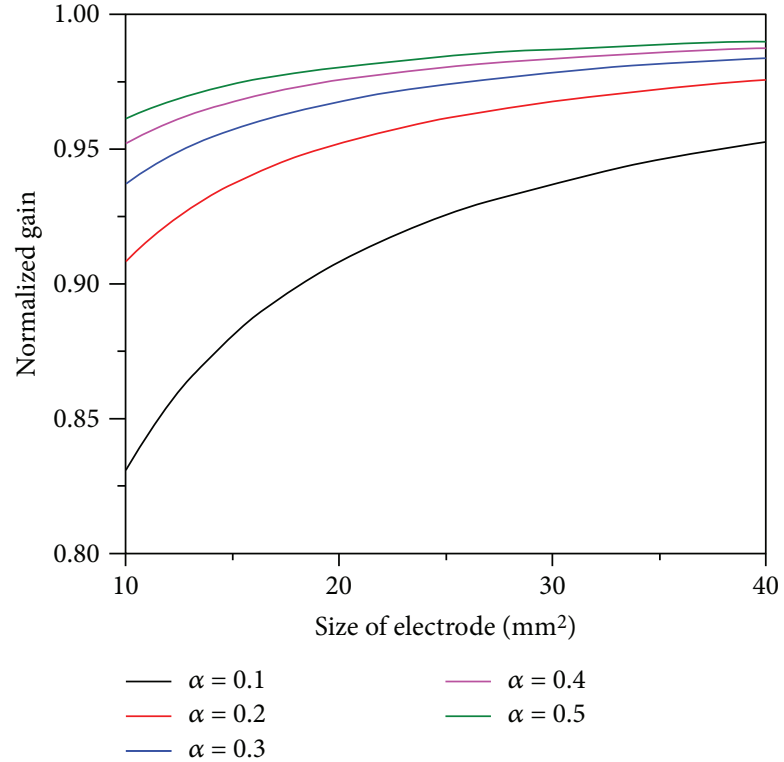

(a)

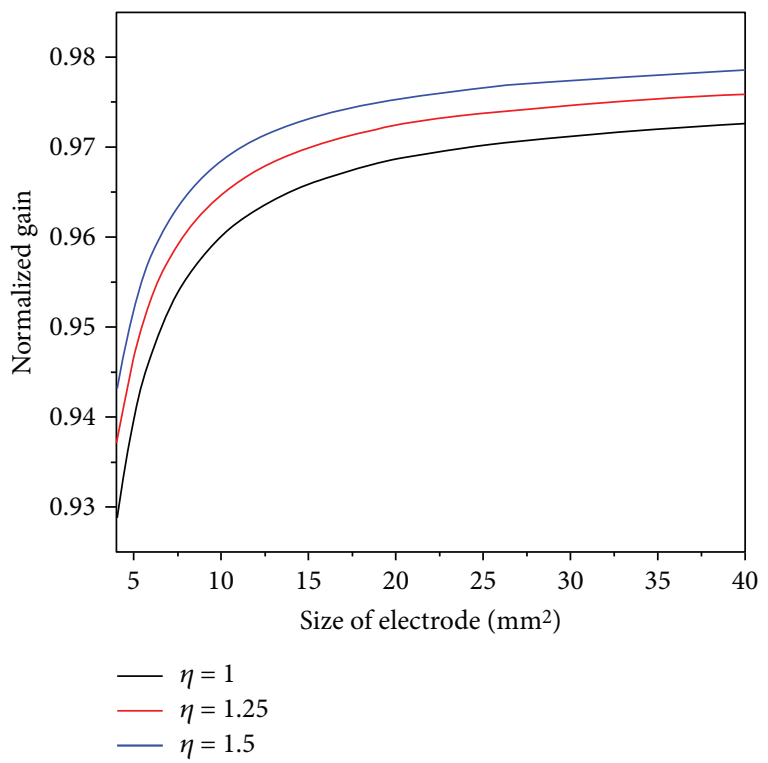

(b)

Figure 4: Output signal gain verse with electrode area with (a) different areal coverage and (b) different height-width ratio.

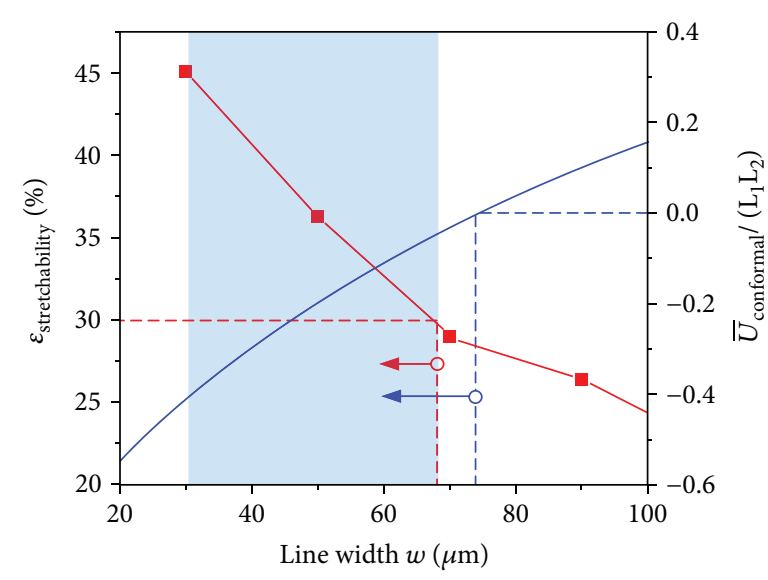

(a)

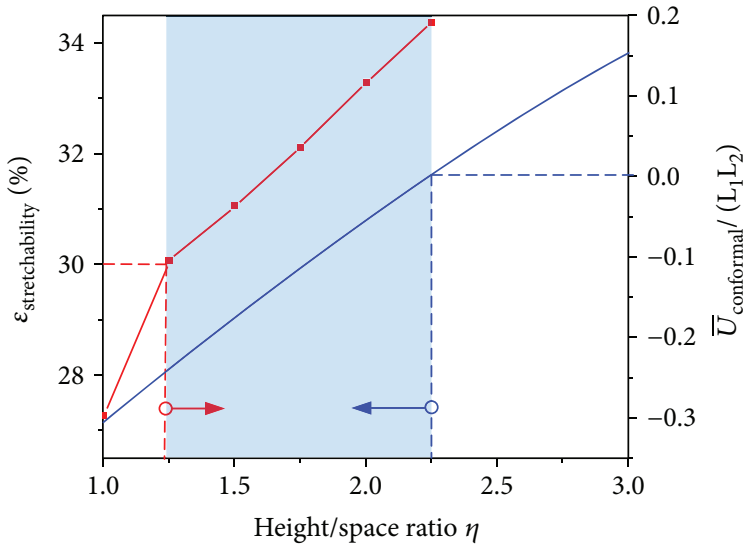

(b)

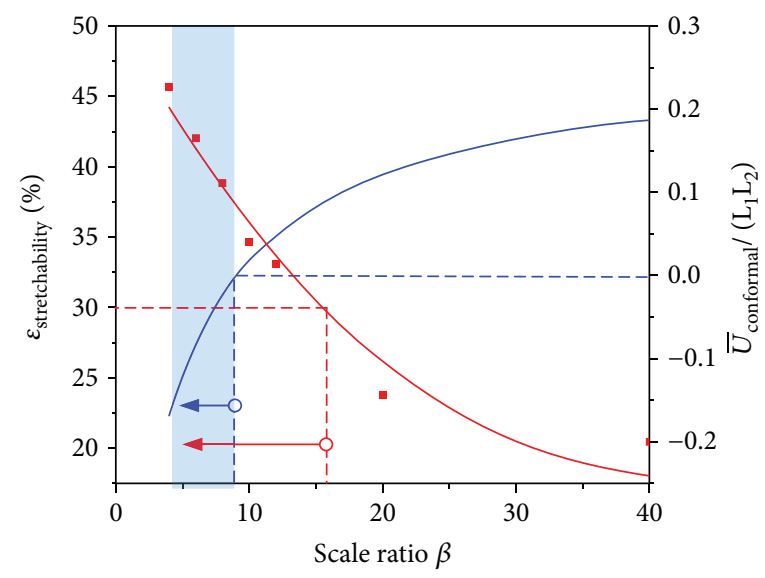

(c)

FiguRE 5: The feasible range of geometric parameters for the stretchability and conformability of the network-shaped electrode: the effects of (a) line width $w$, (b) height-space ratio $\eta$, and (c) scale ratio $\beta$. 

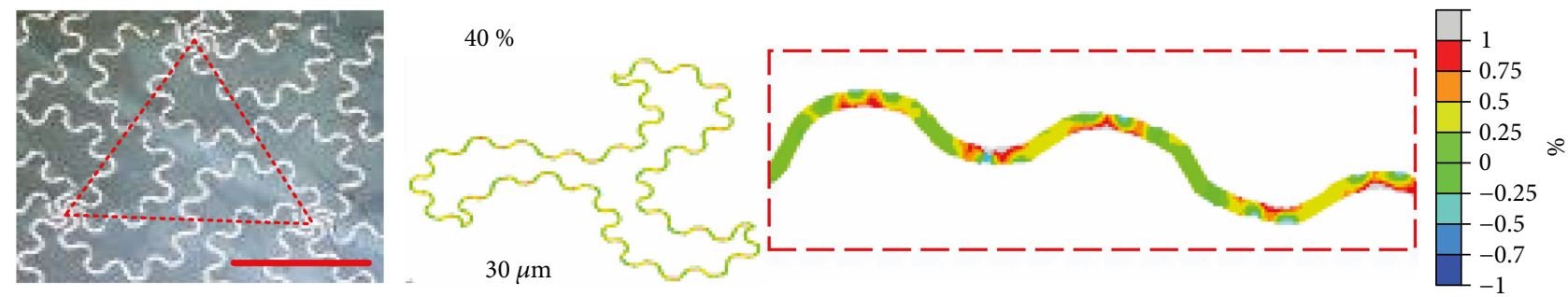

(a)
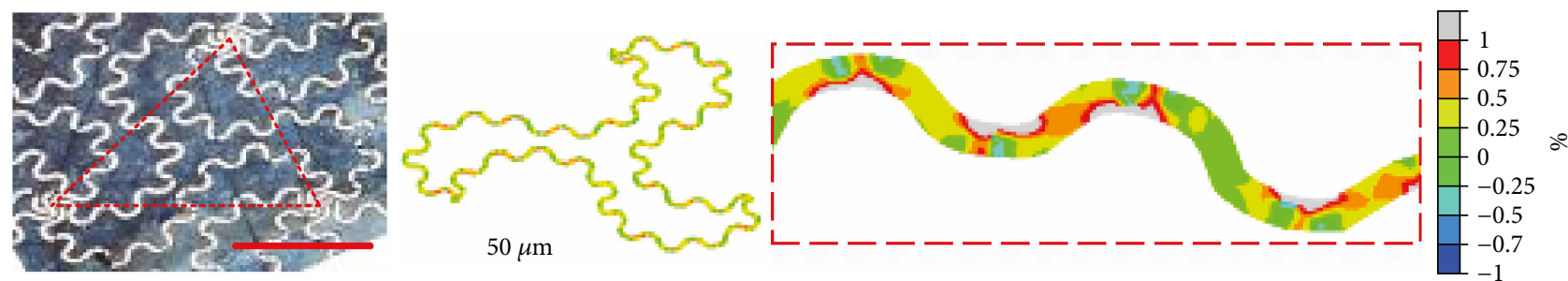

(b)
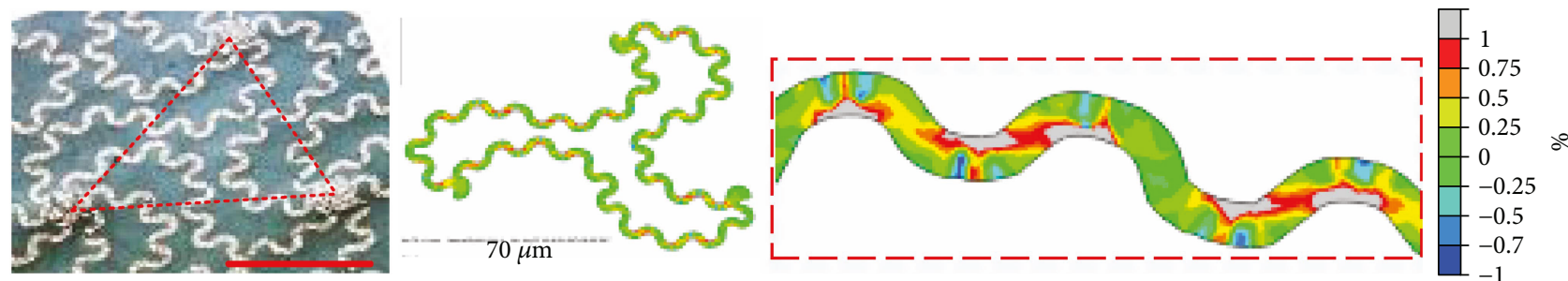

(c)
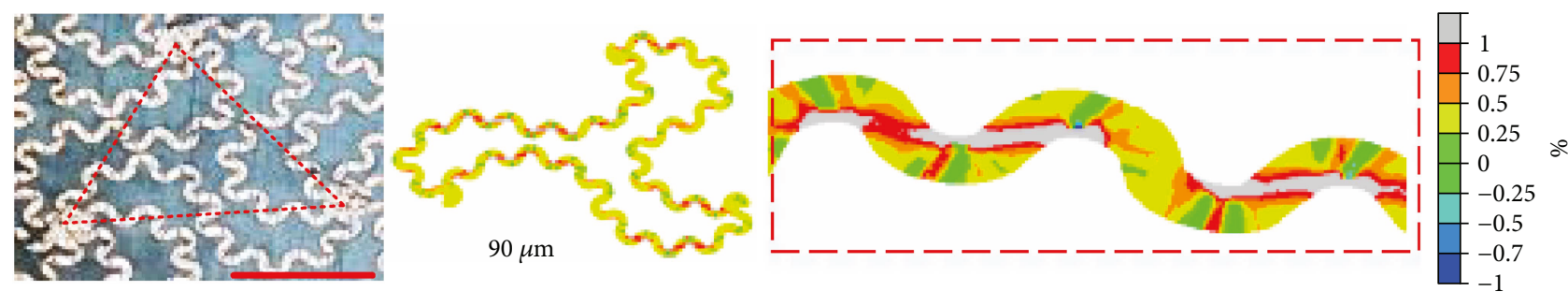

(d)

Figure 6: Experimental and FEM simulation results with different widths in $40 \%$ stretched format: (a) $30 \mu \mathrm{m}$, (b) $50 \mu \mathrm{m}$, (c) $70 \mu \mathrm{m}$, and (d) $90 \mu \mathrm{m}$.

\section{Characterization}

According to feasible range of the geometric parameters, the electrodes with different shapes are prepared to validate the stretchability and conformability of optimization results, where $\beta=8, \eta=1.25$, and $w$ is $30 \mu \mathrm{m}, 50 \mu \mathrm{m}, 70 \mu \mathrm{m}$, and $90 \mu \mathrm{m}$, respectively.

4.1. Stretchability. The network-shaped electrode can match the large deformation of skin surface. The stretchability of the electrode is evaluated by experiments and FEM simulation using the commercial package, Abaqus 6.10. There is no mature theory for the criterion of plastic strain of the thin metal film adhered onto the soft substrate when the thin metal film is stretched. Mostly, FEM simulation and experiment demonstrations are used to validate the stretchability of the thin metal film. The experiment results indicate that the electrodes, with line width $30 \mu \mathrm{m}, 50 \mu \mathrm{m}, 70 \mu \mathrm{m}$, and $90 \mu \mathrm{m}$, are able to be stretched up to $40 \%$ shown in the left frame of Figure 6. The middle frame is the FEM results in $40 \%$, stretched format, and the right frame is the local enlarged graph of the FEM results. The FEM results agree well with the experiments in the respect of structural deformation. The maximum principal strain of network-shaped electrodes increases with the width $w$ from FEM simulation results when stretching the electrodes. It is observed that the electrodes with widths $70 \mu \mathrm{m}$ (Figure 6(c)) and $90 \mu \mathrm{m}$ (Figure $6(\mathrm{~d})$ ) do not satisfy the deformability $(>40 \%)$, as the strain of electrode larger than $1 \%$ is $>1: 4$ in the line width direction. It would reduce the reliability of the electrode with repeated stretching/compressing. The experiment and FEM simulation results indicate that the electrodes with widths $30 \mu \mathrm{m}$ (Figure 6(a)) and $50 \mu \mathrm{m}$ (Figure 6(b)) satisfy the $40 \%$ deformability. 


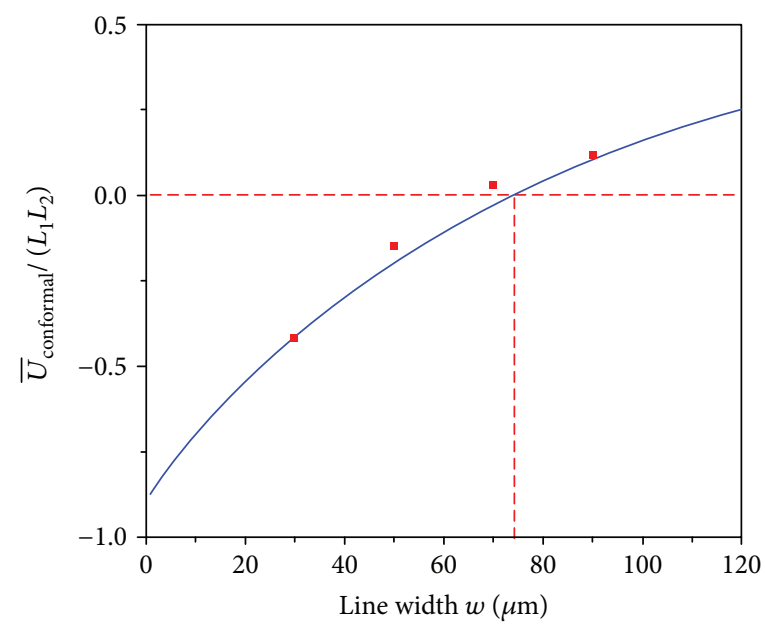

(a)
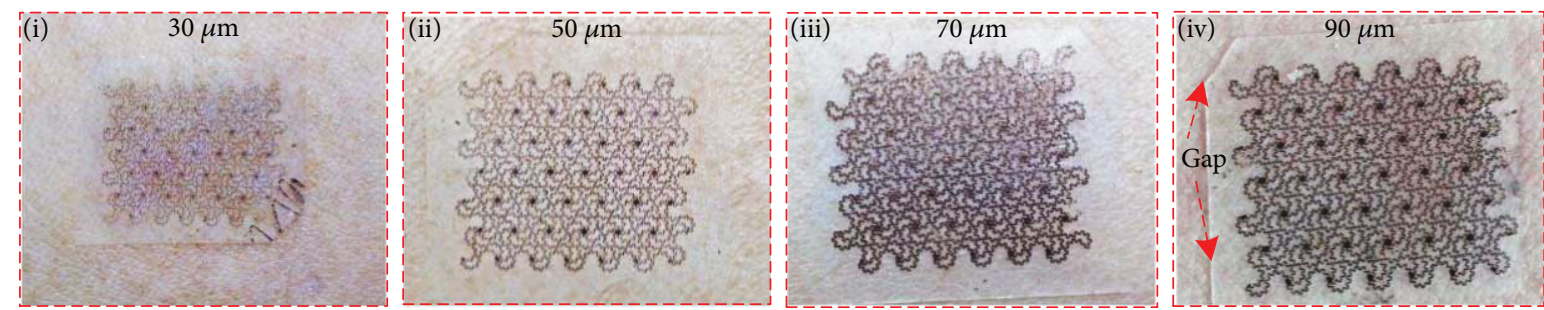

(b)

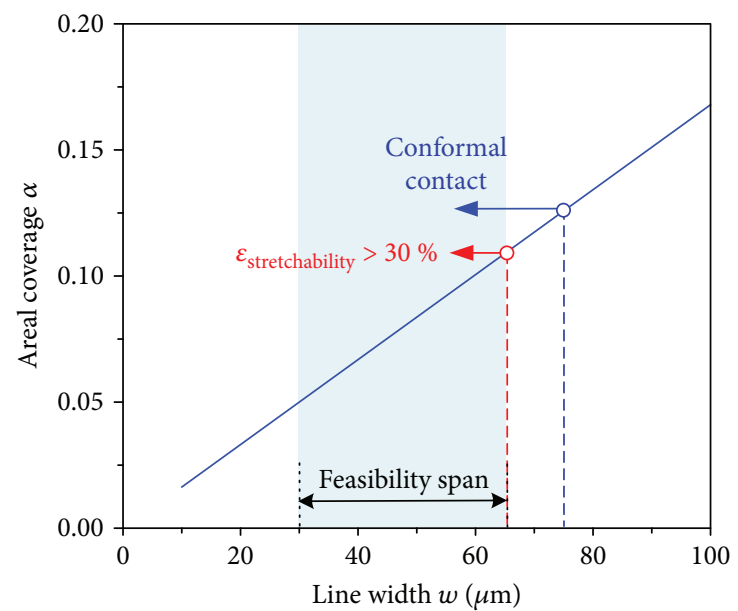

(c)

FIGURE 7: Conformability analysis for the electrodes with different line widths. (a) Theoretical and experiment results of the network-shaped electrode contact with the skin with different line widths. (b) Optical images of the electrodes contact with the skin surface (I) $30 \mu \mathrm{m}$; (II) $50 \mu \mathrm{m}$; (III) $70 \mu \mathrm{m}$; and (IV) $90 \mu \mathrm{m}$. (c) Feasible range of the line width of the electrode with given stretchability and conformability.

4.2. Conformability. Conformal contact between the electrode and the skin improves SNR of EMG signal measurement and reduces the motion artifacts and background noise. The surface electrode adhered onto the skin surface is driven only by van der Waals interactions. Conformal contact occurs when the adhesion energy is larger than the sum of the bending and elastic energy $[19,36,37]$. Figure 7(a) depicts the relationship between the conformability and the line width $w$ via theoretical analysis and experiments, and the total interfacial energy at electrode-skin interface increases with the line width of electrode. The critical line width is $72 \mu \mathrm{m}$ where $U_{\text {total }}=0$, that is, the electrodes with line width smaller than the critical width $72 \mu \mathrm{m}$ can contact with the skin conformally. The scatter data in Figure $7(\mathrm{a})$ are the experimental results with line width $w=30 \mu \mathrm{m}, 50 \mu \mathrm{m}, 70 \mu \mathrm{m}$, and $90 \mu \mathrm{m}$, respectively. The electrodes with $w=30 \mu \mathrm{m}$ and $50 \mu \mathrm{m}$ conformally contact with the skin, and the electrodes with $w=90 \mu \mathrm{m}$ cannot conform with the skin. The experiment data agree well with the theoretical results of (3). Figure 7(b) shows the optical images of the electrode on the skin surface, where the line widths 


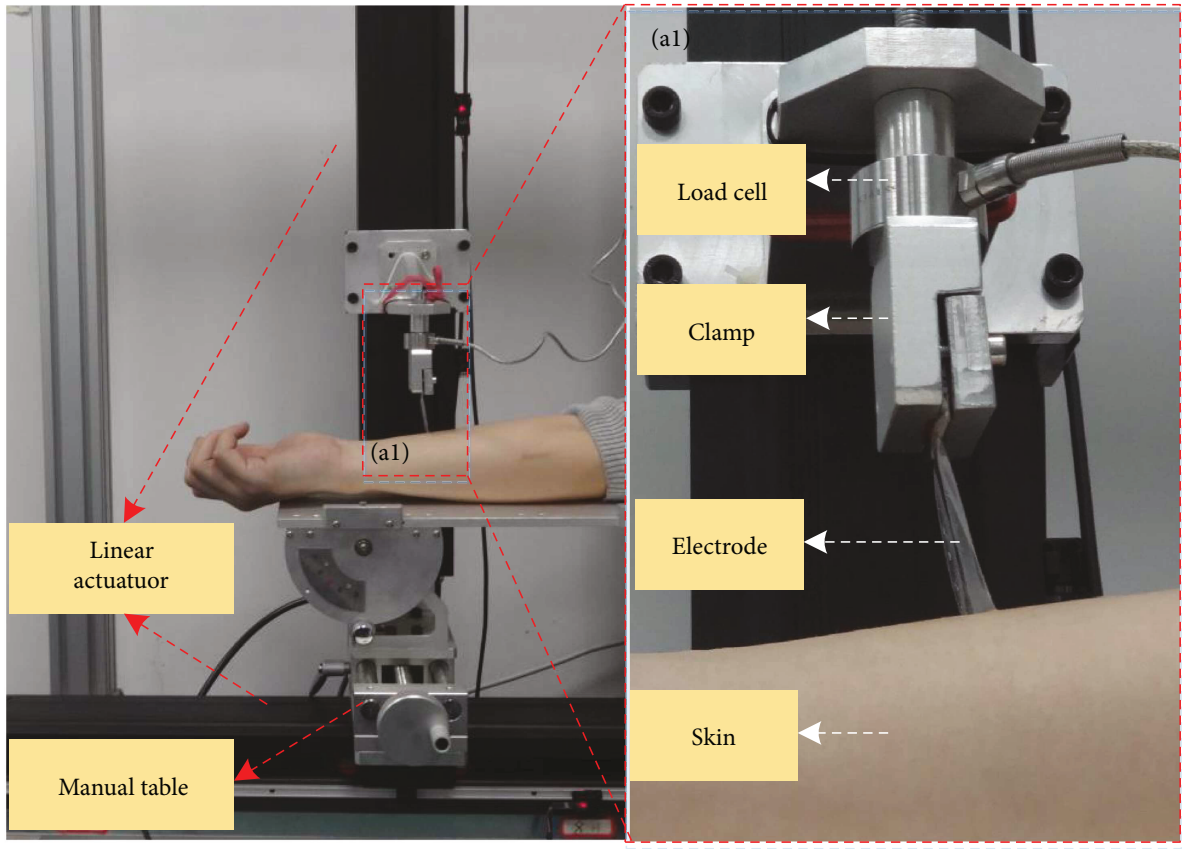

(a)
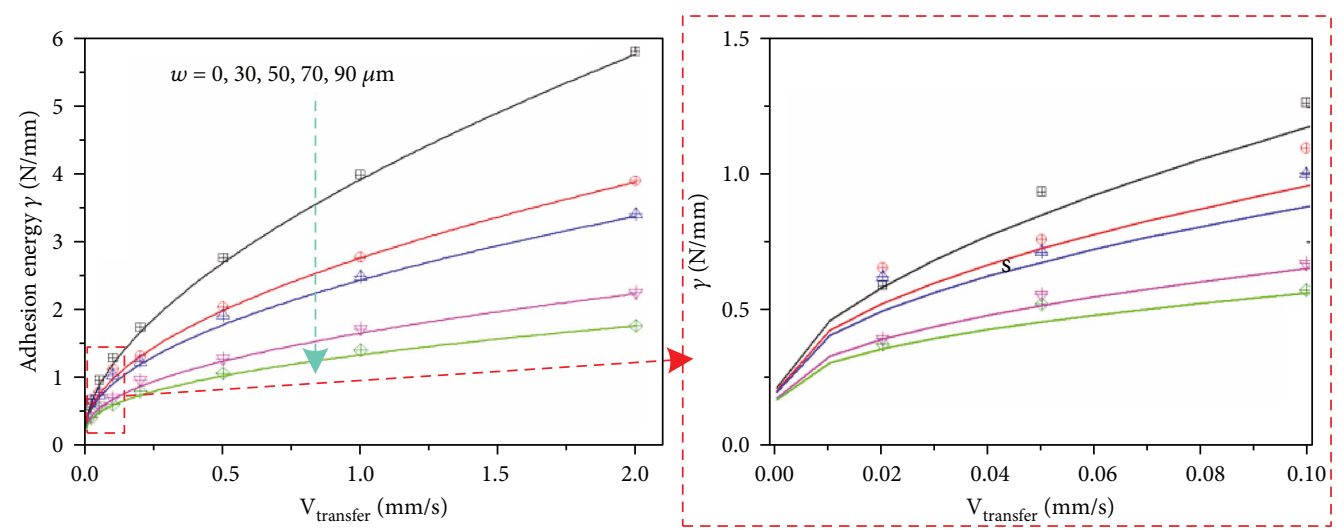

(b)

FIGURE 8: (a) The peeling test experiment platform and the local enlarged graph and (b) experiment data and analysis from the experiment platform at skin-electrode interface with different line widths.

$w$ are (I) $30 \mu \mathrm{m}$, (II) $50 \mu \mathrm{m}$, (III) $70 \mu \mathrm{m}$, and (IV) $90 \mu \mathrm{m}$, respectively. There are gaps between the skin surface and the electrode with line width $90 \mu \mathrm{m}$, which denotes nonconformal contact with the skin surface. The peeling experiments for the stretchable electrodes with different line widths $w=30 \mu \mathrm{m}, 50 \mu \mathrm{m}, 70 \mu \mathrm{m}$, and $90 \mu \mathrm{m}$ are prepared to measure the critical energy at electrode-skin interface via the homemade peeling experiment platform, as shown in Figure 8.

Figure 7 (c) depicts that the areal coverage of the surface electrode increases with the line width $w$. The line width is smaller than $70 \mu \mathrm{m}$ to keep conformal contact with the skin surface shown in Figure $7(\mathrm{a})$. For the stretchability $\varepsilon_{\text {elec- }}$ trode $>30 \%$, the line width is less than $65 \mu \mathrm{m}$ (Figures $5(\mathrm{a})$ and 6). To provide enough areal coverage to collect biological signals, the line width is larger than $30 \mu \mathrm{m}$. Considering the conformability, stretchability, and areal coverage of the surface electrode, the feasible range of line width is in $(30,65) \mu \mathrm{m}$, which agrees well with optimization results in Figure 5.

4.3. EMG Signal Recording. The EMG signals are recorded by the stretchable capacitive electrodes to diagnose neuromuscular disorders and study muscle pain [38]. To minimize the motion artifacts during movement, the electrodes should keep conformal contact with the skin surface when the external load is applied to the electrode. The ground electrode, located in the center of the measurement and reference electrodes (16 $\mathrm{mm}$ apart at center-to-center distance), determines the common-zero potential. A customized PCB is used to collect the EMG data from different parts of the body. It yields analog data, converted to digital signals for recording using LabView software and analysis using MATLAB software. 


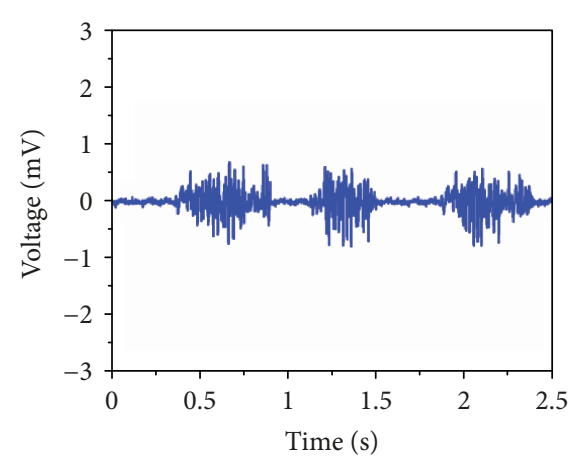

(a)

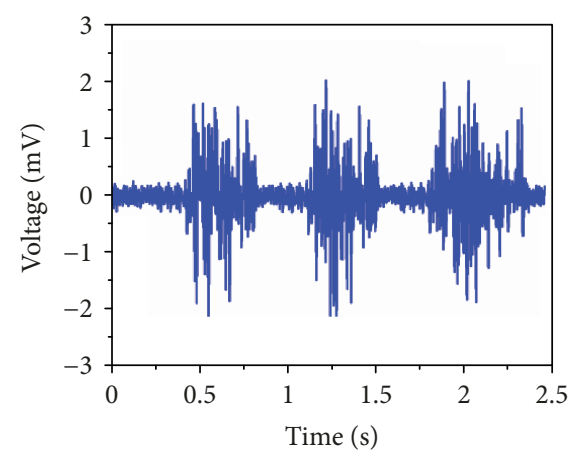

(c)

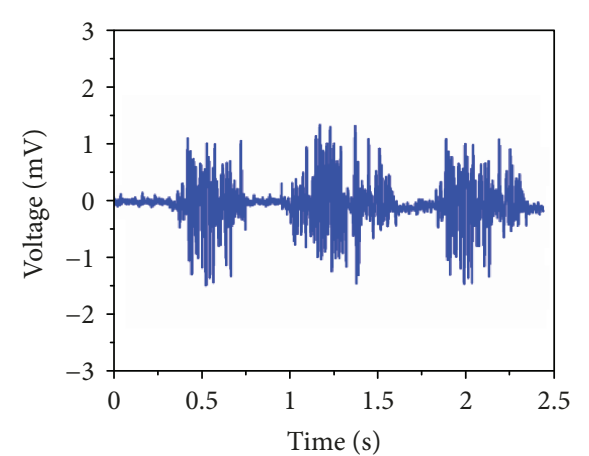

(b)

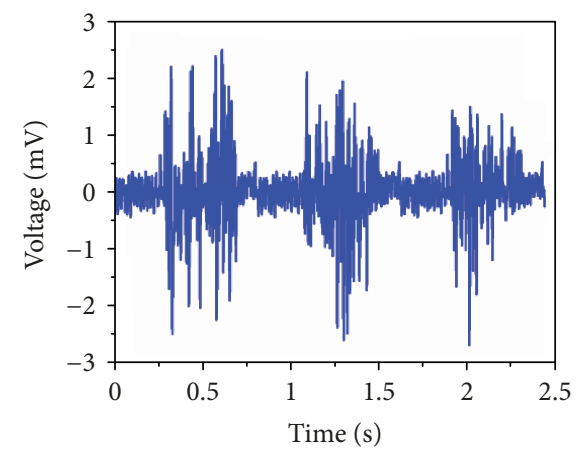

(d)

FIGURE 9: EMG signal from the capacitive electrode with different line widths. (a) $w=30 \mu \mathrm{m}$; (b) $w=50 \mu \mathrm{m}$; (c) $w=70 \mu \mathrm{m}$; and (d) $w=90 \mu \mathrm{m}$.

Figure 9 depicts the EMG signal from the capacitive electrode with different line widths $w=30 \mu \mathrm{m}, 50 \mu \mathrm{m}, 70 \mu \mathrm{m}$, and $90 \mu \mathrm{m}$. The EMG signal is clearly distinguishable from the baseline noise, and it has potential in clinics for diagnosis of neuromuscular disorders. As shown in I-II of Figure 7(b), the electrodes with line width $30 \mu \mathrm{m}$ and $50 \mu \mathrm{m}$ keep conformal contact with the skin surface and also achieve the smaller background noise. The background noise from the electrode with line width $w=90 \mu \mathrm{m}$ is larger as it cannot conformally contact with skin as shown in (Figure 7(b) (IV)). Based on the EMG and background noise signal from the electrodes with different line widths, SNR from electrodes with line width $50 \mu \mathrm{m}$ is larger than others.

4.4. Electrical Performance. The electrical performances (capacitance $C_{\mathrm{E}}$ at skin-electrode interface, output gain, SNR, and background noise) are related to the geometry parameters of the electrode $[39,40]$. Capacitance $C_{E}$ determines SNR through its effect on the gain of the preamplifier. As expected, the output gain $G$ is also improved as the $C_{E}$ is increased with increasing effective area of the electrodes [41]. Figure 10(a) depicts the experimental and theoretical results of the output gain and the area of the electrode at areal coverage $\alpha=0.2$. The scatter points in Figure 10(a) are the experimental results with area $=20 \mathrm{~mm}^{2}, 30 \mathrm{~mm}^{2}$, and $40 \mathrm{~mm}^{2}$, respectively. It is seen that experimental results agree well with the theoretical results. The output gain of the electrode increases with the area of the electrode. Figure 10(b) depicts SNR from the capacitive EMG electrode with line width $w=30 \mu \mathrm{m}, 50 \mu \mathrm{m}, 70 \mu \mathrm{m}$, and $90 \mu \mathrm{m}$, respectively. Signal voltage and noise voltage represent the amplitude of the EMG signal and background noise signal. The histogram shows that SNR from the electrode with line width $w=50 \mu \mathrm{m}$ is larger than line width $w=30 \mu \mathrm{m}, 70 \mu \mathrm{m}$, and $90 \mu \mathrm{m}$. Figure 10(c) depicts the skin-electrode interface contact impedance decreases with the increasing scanning frequency with the area $24 \mathrm{~mm}^{2}$ and $40 \mathrm{~mm}^{2}$. Larger effective contact area provides smaller contact impedance which indicates that area coverage would play important part in output gain of the electrode with same area. Figure 10(d) depicts the leakage current from the capacitive electrodes is rather small compared to the conventional medical biological electrodes. It shows that the current is below $0.2 \mathrm{~mA}$ when $10 \mathrm{~mA}$ passes through the electrodes. It is helpful to protect the soft biological tissues during biological signal monitoring.

4.5. Motion Artifacts. Conformability at the electrode-skin interface reduces the motion artifacts due to the body motion, and motion artifacts exert important effects on the susceptibility and stability of biological signal recordings via capacitive electrodes. Compared to EMG signals, the features of ECG are very oblivious with "PQRST," especially in the case the biological signals are contaminated by the external factors [42]. Herein, the ECG signals are used to illustrate the stability and robustness of the surface electrode for biological signal recordings. Figure 11 depicts that the ECG signals are recorded from the electrodes placed onto the surface of left chest with different line widths $w=30 \mu \mathrm{m}, 50 \mu \mathrm{m}, 70 \mu \mathrm{m}$, and $90 \mu \mathrm{m}$ in the case of external loads (such as running and body swing). Figures 11(a) and 11(b) show that the electrodes with line widths $w=30$ and $50 \mu \mathrm{m}$ realize the continuous 


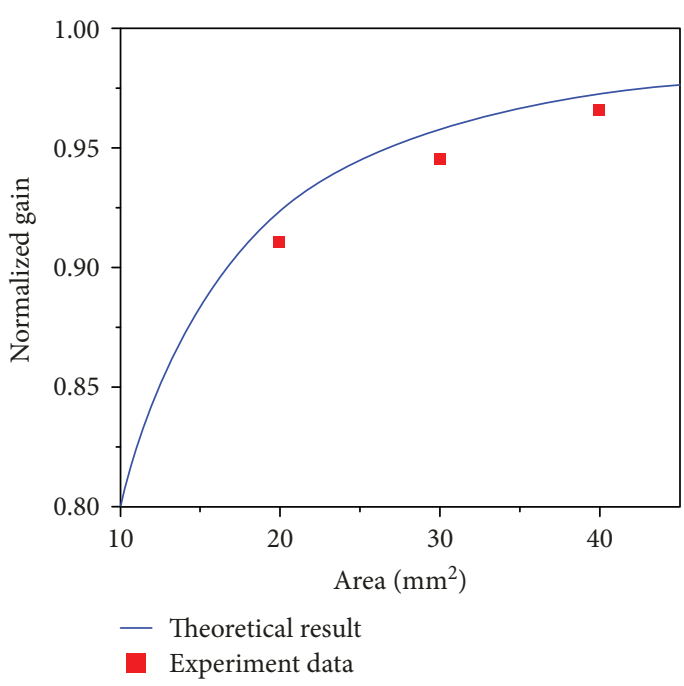

(a)

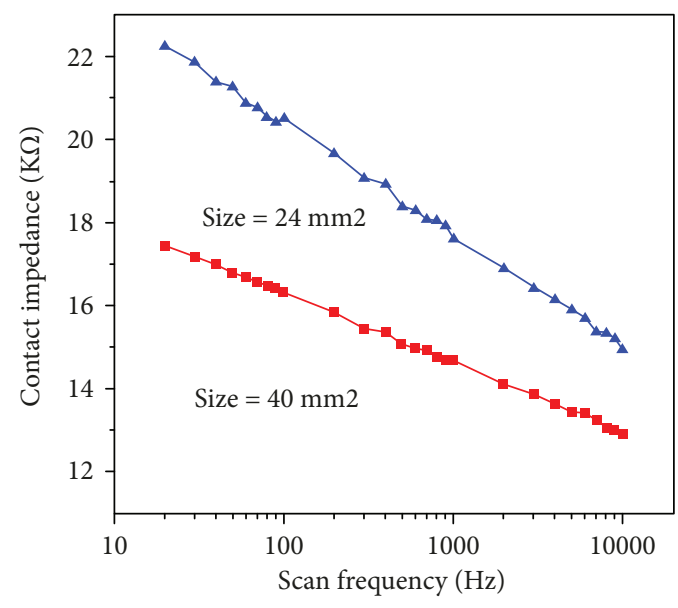

(c)

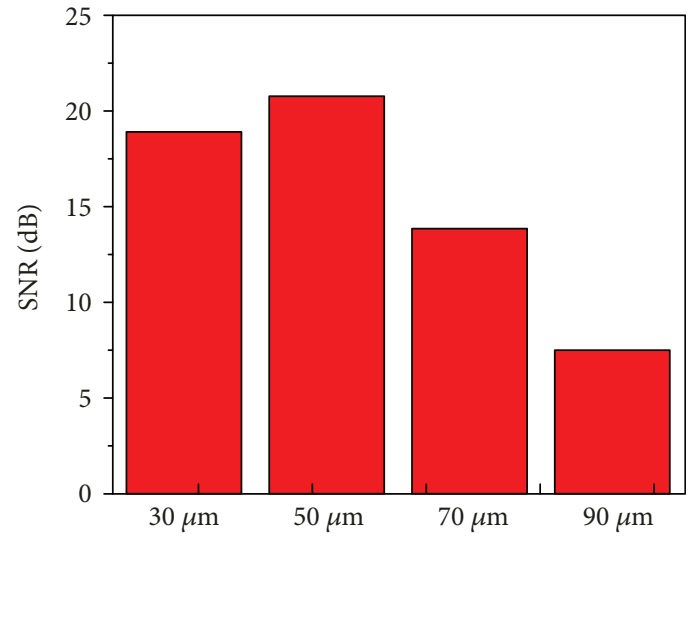

(b)

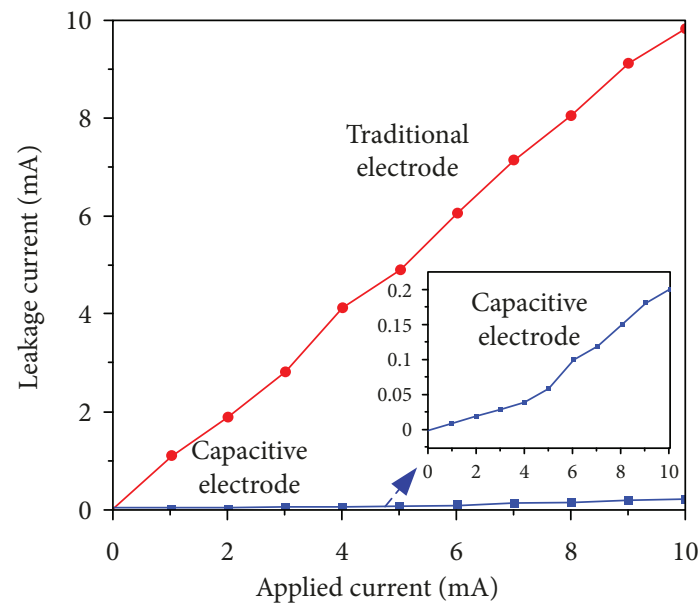

(d)

FIGURE 10: Electrical performance of the capacitive biological electrode. (a) The experimental and theoretical results between the output gain and the area. (b) SNR from the capacitive biological electrode with different line widths. (c) Contact impedance versus with the scanning frequency. (d) The leakage current at the skin/electrode interface.

ECG signal recordings, and they still record the ECG signals when body runs. Figures 5(c) and 5(d) depict that ECG signals are contaminated as the artifacts induced by body movements by the electrode with line width $w=70 \mu \mathrm{m}$ and $90 \mu \mathrm{m}$. Stretchable electrodes with line widths $70 \mu \mathrm{m}$ and $90 \mu \mathrm{m}$ do not contact with skin surface conformally, which agrees well with the optimized structural results in Figure 7. It is clearly shown that the optimized electrode with line $w=50 \mu \mathrm{m}$ records ECG signal continuously and stably as it contacts with skin conformally.

\section{Conclusion}

Electromechanical optimization method for the stretchable capacitive electrodes with self-similar configuration was studied to realize excellent mechanical (large deformation $>30 \%$ and conformal contact with skin) adaptability and simultaneously improve the electrical performance (high SNR and signal stability). Feasible range of the geometry parameters of the 2nd-order self-similar serpentine structure was determined by solving the optimization model. The stretchability of the electrode was validated by experiment and FEM simulation. Peeling test experiments were made to verify the theoretical results for the conformability at electrode-skin interface. Electrode with line width $w=50 \mu \mathrm{m}$ performs better for considering the stretchability, conformability, EMG, and ECG signal recordings. The proposed optimization model has general utility for future work in stretchable electronics, especially those demand high stretchability, and high filling factors of active devices.

\section{Appendix}

\section{A. Solving Process Chart for the Optimization Model}

The electrical performance of the surface electrode is related to the geometric parameters $\eta, \beta$, and $w$. It is 


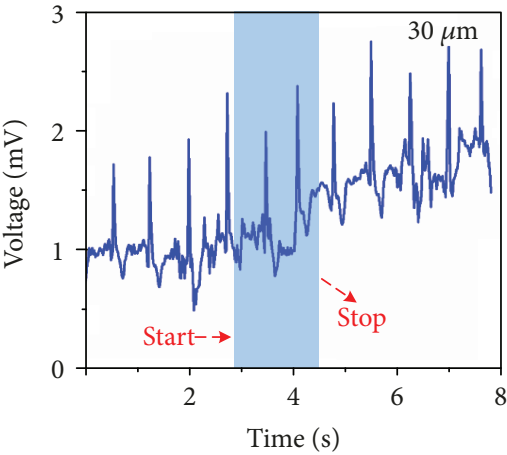

(a)

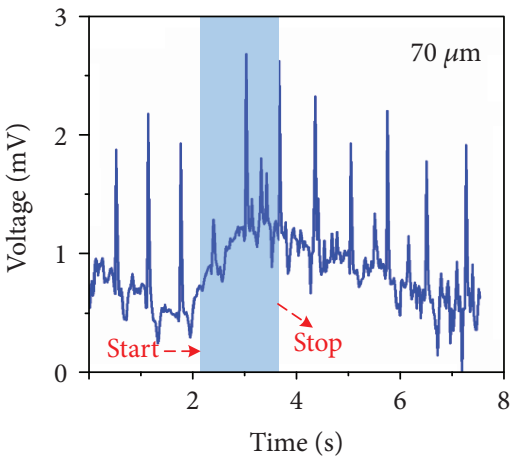

(c)

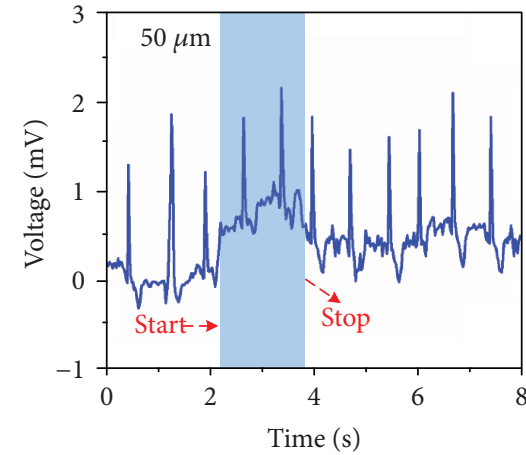

(b)

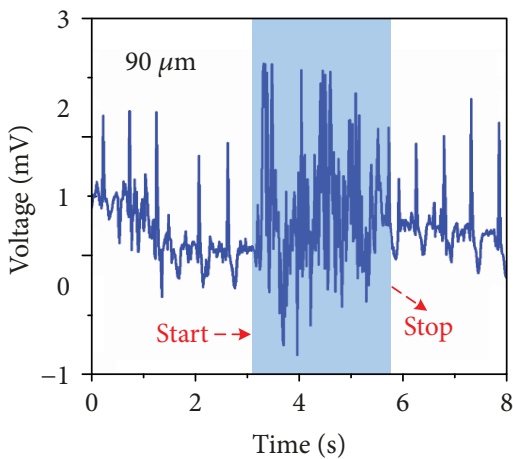

(d)

FIGURE 11: ECG recordings from network-shaped electrodes with different line width. (a) $w=30 \mu \mathrm{m}$; (b) $w=50 \mu \mathrm{m}$; (c) $w=70 \mu \mathrm{m}$; and (d) $w=90 \mu \mathrm{m}$.

difficult to solve the optimization model (2), (3), (4), and (5) with the coupling mechanical and electrical properties of the surface electrode. Three-stage solving method is proposed to solve the optimization model. Figure 2 depicts the detailed solving process for optimized structural design of the electrodes, mainly including three steps: parameter optimization of the microinterconnect unit, geometry size and layout of surface electrode, and structure optimization of surface electrode.

\section{B. Experiment Setup for Adhesion Energy Measurement}

The homemade experimental platform with motion and velocity adjusted part is shown in Figure 8(a). The peeling velocity is adjusted from $0.05 \mathrm{~mm} / \mathrm{s}-100 \mathrm{~mm} / \mathrm{s}$, and the peel force is obtained using load cell (222-N LCM300, FUTEK, USA) with an accuracy of $1 \mathrm{mN}$. Test samples of the electrodes with different line widths $w=$ $30 \mu \mathrm{m}, 50 \mu \mathrm{m}, 70 \mu \mathrm{m}$, and $90 \mu \mathrm{m}$ are prepared for the peeling experiments which are used to measure the critical energy at electrode-skin interface. The peeling force per unit has been measured for the electrode peeling from skin surface by the peeling experiment platform with the peeling velocity $v_{\text {peel }}=0.02,0.05,0.1 \mathrm{~mm} / \mathrm{s}$, $0.2 \mathrm{~mm} / \mathrm{s}, 0.5 \mathrm{~mm} / \mathrm{s}, 1 \mathrm{~mm} / \mathrm{s}$, and $2 \mathrm{~mm} / \mathrm{s}$. It is plotted in Figure 8 (b) with scatter point, and exponential function is used to fit the scatter data.

\section{Data Availability}

The data used to support the findings of this study are available from the corresponding author upon request.

\section{Conflicts of Interest}

The authors declare that they have no conflicts of interest.

\section{Acknowledgments}

The authors acknowledge supports from the National Natural Science Foundation of China (51635007), Special Project of Technology Innovation of Hubei Province (2017AAA002), and Program for HUST Academic Frontier Youth Team. The authors would also like to thank Flexible Electronics Manufacturing Laboratory in Comprehensive Experiment Center for Advanced Manufacturing and Equipment Technology for fabricating surface electromyography electrodes.

\section{References}

[1] R. Cardu, P. H. W. Leong, C. T. Jin, and A. McEwan, "Electrode contact impedance sensitivity to variations in geometry," Physiological Measurement, vol. 33, no. 5, pp. 817-830, 2012.

[2] M. B. Weil, M. Oehler, M. Schilling, and L. S. Maier, "First clinical evaluation of a novel capacitive ECG system in 
patients with acute myocardial infarction," Clinical Research in Cardiology, vol. 101, no. 3, pp. 165-174, 2012.

[3] H. Li, K. Kawashima, K. Tadano, S. Ganguly, and S. Nakano, "Achieving haptic perception in forceps' manipulator using pneumatic artificial muscle," IEEE/ASME Transactions on Mechatronics, vol. 18, no. 1, pp. 74-85, 2013.

[4] Y. Huang, Y. Ding, J. Bian et al., "Hyper-stretchable selfpowered sensors based on electrohydrodynamically printed, self-similar piezoelectric nano/microfibers," Nano Energy, vol. 40, pp. 432-439, 2017.

[5] M. Ferre, I. Galiana, R. Wirz, and N. Tuttle, "Haptic device for capturing and simulating hand manipulation rehabilitation," IEEE/ASME Transactions on Mechatronics, vol. 16, no. 5, pp. 808-815, 2011.

[6] D. N. Mathias, S.-I. Kim, J.-S. Park, Y.-H. Joung, and W. S. Choi, "Electrode characteristics of non-contact electrocardiographic measurement," Transactions on Electrical and Electronic Materials, vol. 16, no. 1, pp. 42-45, 2015.

[7] D. H. Kim, N. Lu, R. Ma et al., "Epidermal electronics," Science, vol. 333, no. 6044, pp. 838-843, 2011.

[8] J.-W. Jeong, W.-H. Yeo, A. Akhtar et al., "Materials and optimized designs for human-machine interfaces via epidermal electronics," Advanced Materials, vol. 25, no. 47, pp. 68396846, 2013.

[9] D.-H. Kim and J. A. Rogers, "Stretchable electronics: materials strategies and devices," Advanced Materials, vol. 20, no. 24, pp. 4887-4892, 2008.

[10] M. L. Hammock, A. Chortos, B. C.-K. Tee, J. B.-H. Tok, and Z. Bao, "25th anniversary article: the evolution of electronic skin (e-skin): a brief history, design considerations, and recent progress," Advanced Materials, vol. 25, no. 42, pp. 5997-6038, 2013.

[11] H. Bahrami, S. A. Mirbozorgi, L. A. Rusch, and B. Gosselin, "Biological channel modeling and implantable UWB antenna design for neural recording systems," IEEE Transactions on Biomedical Engineering, vol. 62, no. 1, pp. 88-98, 2015.

[12] H. Zhang, W. Pei, Y. Chen et al., "A motion interferenceinsensitive flexible dry electrode," IEEE Transactions on Biomedical Engineering, vol. 63, no. 6, pp. 1136-1144, 2016.

[13] S. Han, M. K. Kim, B. Wang, D. S. Wie, S. Wang, and C. H. Lee, "Mechanically reinforced skin-electronics with networked nanocomposite elastomer," Advanced Materials, vol. 28, no. 46, pp. 10257-10265, 2016.

[14] Y. Wang, L. Wang, T. Yang et al., "Wearable and highly sensitive graphene strain sensors for human motion monitoring," Advanced Functional Materials, vol. 24, no. 29, pp. 46664670, 2014.

[15] W. G. Bae, D. Kim, M. K. Kwak, L. Ha, S. M. Kang, and K. Y. Suh, "Enhanced skin adhesive patch with modulus-tunable composite micropillars," Advanced Healthcare Materials, vol. 2, no. 1, pp. 109-113, 2013.

[16] A. A. Gopalai and S. M. N. Arosha Arosha Senanayake, "A wearable real-time intelligent posture corrective system using vibrotactile feedback," IEEE/ASME Transactions on Mechatronics, vol. 16, no. 5, pp. 827-834, 2011.

[17] S. H. Jeong, S. Zhang, K. Hjort, J. Hilborn, and Z. Wu, "PDMSbased elastomer tuned soft, stretchable, and sticky for epidermal electronics," Advanced Materials, vol. 28, no. 28, pp. 5830-5836, 2016.

[18] J. Y. Sun, C. Keplinger, G. M. Whitesides, and Z. Suo, "Ionic skin," Advanced Materials, vol. 26, no. 45, pp. 7608-7614, 2014.
[19] W. Dong, L. Xiao, C. Zhu et al., "Theoretical and experimental study of 2D conformability of stretchable electronics laminated onto skin," Science China Technological Sciences, vol. 60, no. 9, pp. 1415-1422, 2017.

[20] W. Dong, C. Zhu, D. Ye, and Y. Huang, "Optimal design of self-similar serpentine interconnects embedded in stretchable electronics," Applied Physics A, vol. 123, no. 6, p. 428, 2017.

[21] Y. Huang, W. Dong, T. Huang et al., "Self-similar design for stretchable wireless LC strain sensors," Sensors and Actuators A: Physical, vol. 224, pp. 36-42, 2015.

[22] R. Li, M. Li, Y. Su, J. Song, and X. Ni, “An analytical mechanics model for the island-bridge structure of stretchable electronics," Soft Matter, vol. 9, no. 35, p. 8476, 2013.

[23] S. Xu, Y. Zhang, J. Cho et al., "Stretchable batteries with selfsimilar serpentine interconnects and integrated wireless recharging systems," Nature Communications, vol. 4, no. 1, 2013.

[24] S. Xu, Y. Zhang, L. Jia et al., "Soft microfluidic assemblies of sensors, circuits, and radios for the skin," Science, vol. 344, no. 6179 , pp. 70-74, 2014.

[25] K. I. Jang, H. U. Chung, S. Xu et al., "Soft network composite materials with deterministic and bio-inspired designs," Nature Communications, vol. 6, no. 1, p. 6566, 2015.

[26] J. A. Fan, W. H. Yeo, Y. Su et al., "Fractal design concepts for stretchable electronics," Nature Communications, vol. 5, no. 1, p. 3266, 2014.

[27] J. W. Jeong, M. K. Kim, H. Cheng et al., "Capacitive epidermal electronics for electrically safe, long-term electrophysiological measurements," Advanced Healthcare Materials, vol. 3, no. 5, pp. 642-648, 2014.

[28] Q. Ma and Y. Zhang, "Mechanics of fractal-inspired horseshoe microstructures for applications in stretchable electronics," Journal of Applied Mechanics, vol. 83, no. 11, article 111008, 2016.

[29] Y. Su, S. Wang, Y. A. Huang et al., "Elasticity of fractal inspired interconnects," Small, vol. 11, no. 3, pp. 367-373, 2015.

[30] Y. Sun and X. B. Yu, "Capacitive biopotential measurement for electrophysiological signal acquisition: a review," IEEE Sensors Journal, vol. 16, no. 9, pp. 2832-2853, 2016.

[31] S. Wang, M. Li, J. Wu et al., "Mechanics of epidermal electronics," Journal of Applied Mechanics, vol. 79, no. 3, article 31022, 2012.

[32] H. Fu, S. Xu, R. Xu et al., "Lateral buckling and mechanical stretchability of fractal interconnects partially bonded onto an elastomeric substrate," Applied Physics Letters, vol. 106, no. 9, article 091902, 2015.

[33] Y. Zhang, S. Xu, H. Fu et al., "Buckling in serpentine microstructures and applications in elastomer-supported ultrastretchable electronics with high areal coverage," Soft Matter, vol. 9, no. 33, pp. 8062-8070, 2013.

[34] Y. Su, J. Wu, Z. Fan et al., "Postbuckling analysis and its application to stretchable electronics," Journal of the Mechanics and Physics of Solids, vol. 60, no. 3, pp. 487-508, 2012.

[35] J. W. Lee, R. Xu, S. Lee et al., "Soft, thin skin-mounted power management systems and their use in wireless thermography," Proceedings of the National Academy of Sciences, vol. 113, no. 22, pp. 6131-6136, 2016.

[36] J. Song, H. Jiang, W. M. Choi, D. Y. Khang, Y. Huang, and J. A. Rogers, "An analytical study of two-dimensional buckling of 
thin films on compliant substrates," Journal of Applied Physics, vol. 103, no. 1, article 014303, 2008.

[37] H. Cheng and S. Wang, "Mechanics of interfacial delamination in epidermal electronics systems," Journal of Applied Mechanics, vol. 81, no. 4, article 44501, 2014.

[38] X. Yu, Z. Yu, W. Pang, M. Li, and L. Wu, "An improved EMDbased dissimilarity metric for unsupervised linear subspace learning," Complexity, vol. 2018, Article ID 8917393, 24 pages, 2018.

[39] M. A. Yokus and J. S. Jur, "Fabric-based wearable dry electrodes for body surface biopotential recording," IEEE Transactions on Biomedical Engineering, vol. 63, no. 2, pp. 423-430, 2016.

[40] D. C. Deno, H. J. Sih, S. P. Miller, L. R. Teplitsky, and R. Kuenzi, "Measurement of electrical coupling between cardiac ablation catheters and tissue," IEEE Transactions on Biomedical Engineering, vol. 61, no. 3, pp. 765-774, 2014.

[41] Y. Lu, Z. Ju, Y. Liu, Y. Shen, and H. Liu, “Time series modeling of surface EMG based hand manipulation identification via expectation maximization algorithm," Neurocomputing, vol. 168, pp. 661-668, 2015.

[42] S. Poungponsri and X.-H. Yu, "An adaptive filtering approach for electrocardiogram (ECG) signal noise reduction using neural networks," Neurocomputing, vol. 117, pp. 206-213, 2013. 


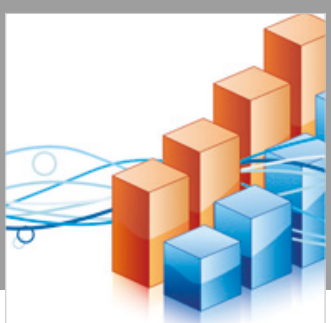

Advances in

Operations Research

\section{-n-m}
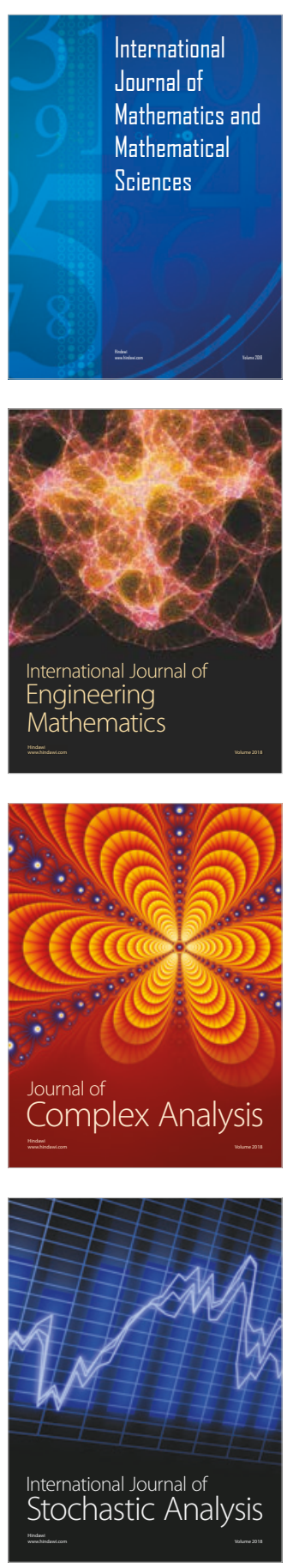
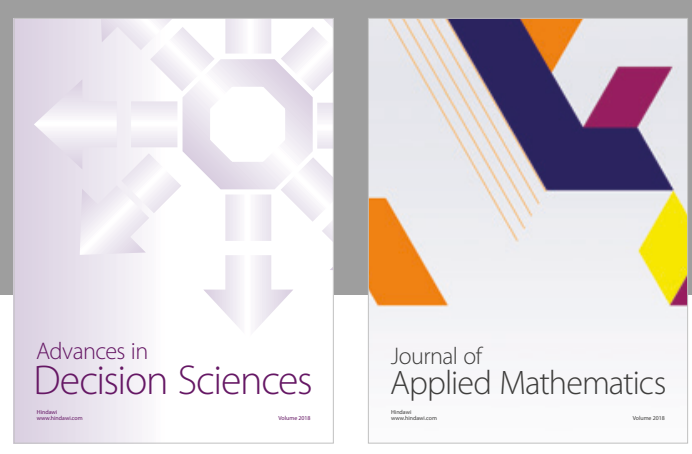

Journal of

Applied Mathematics
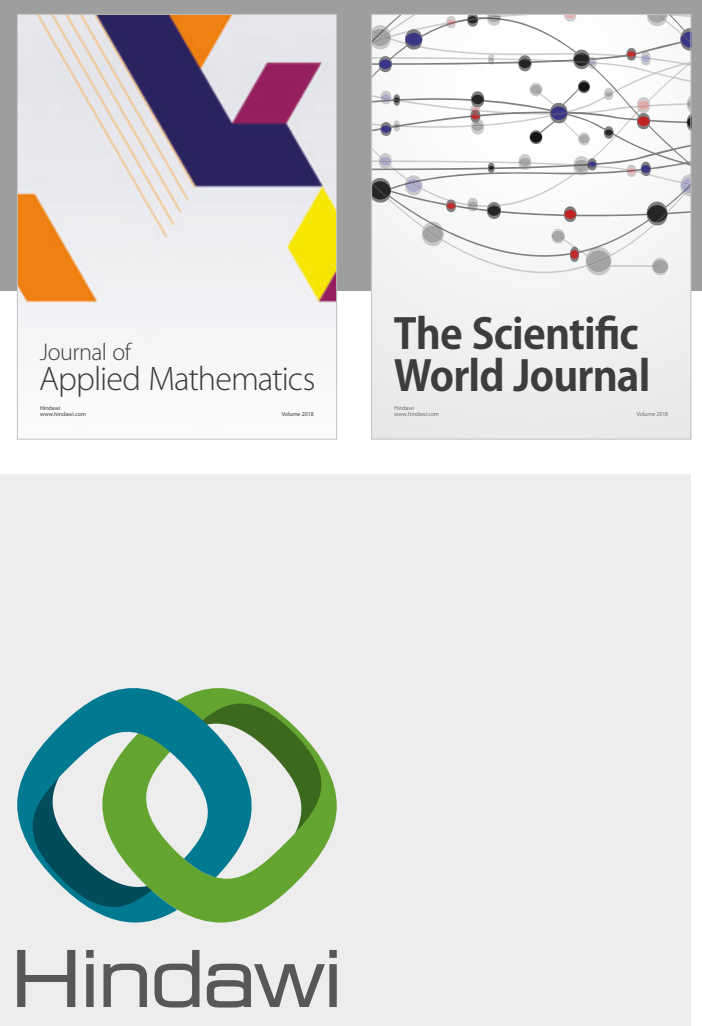

Submit your manuscripts at

www.hindawi.com

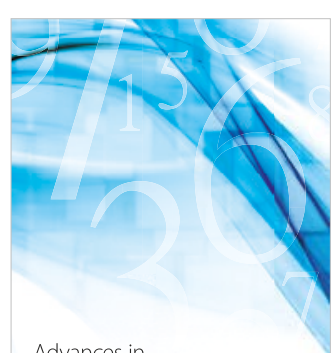

Advances in
Numerical Analysis
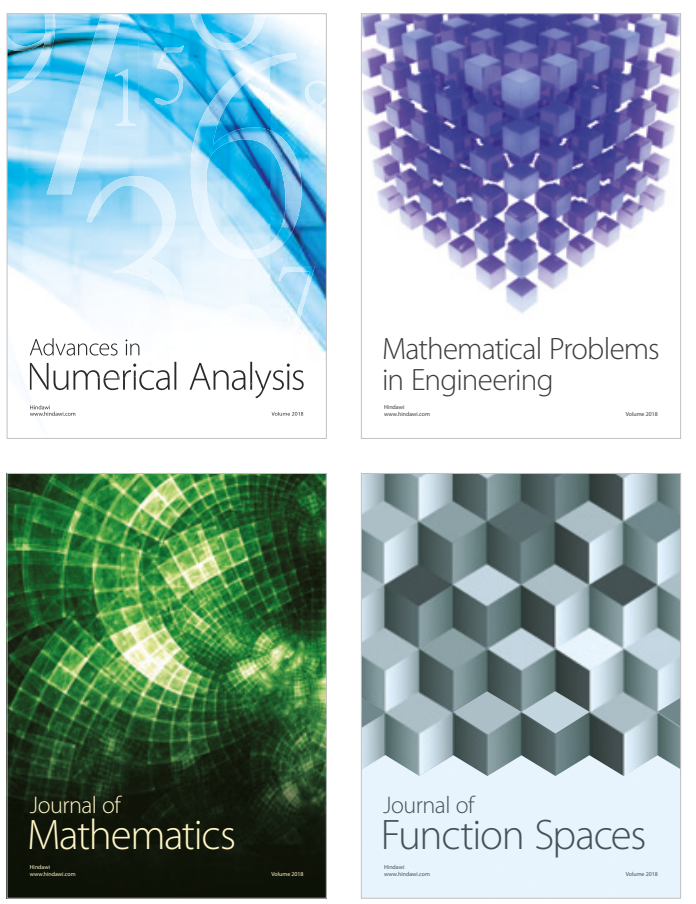

Mathematical Problems in Engineering

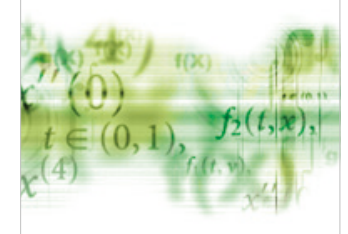

International Journal of

Differential Equations

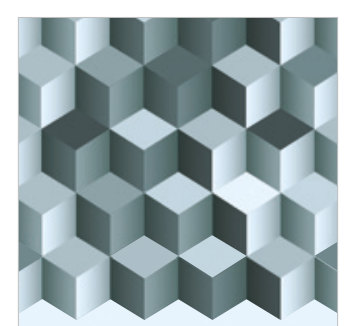

Journal of

Function Spaces
The Scientific

World Journal

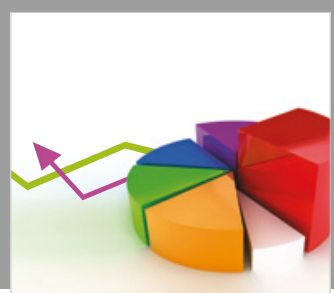

Journal of

Probability and Statistics
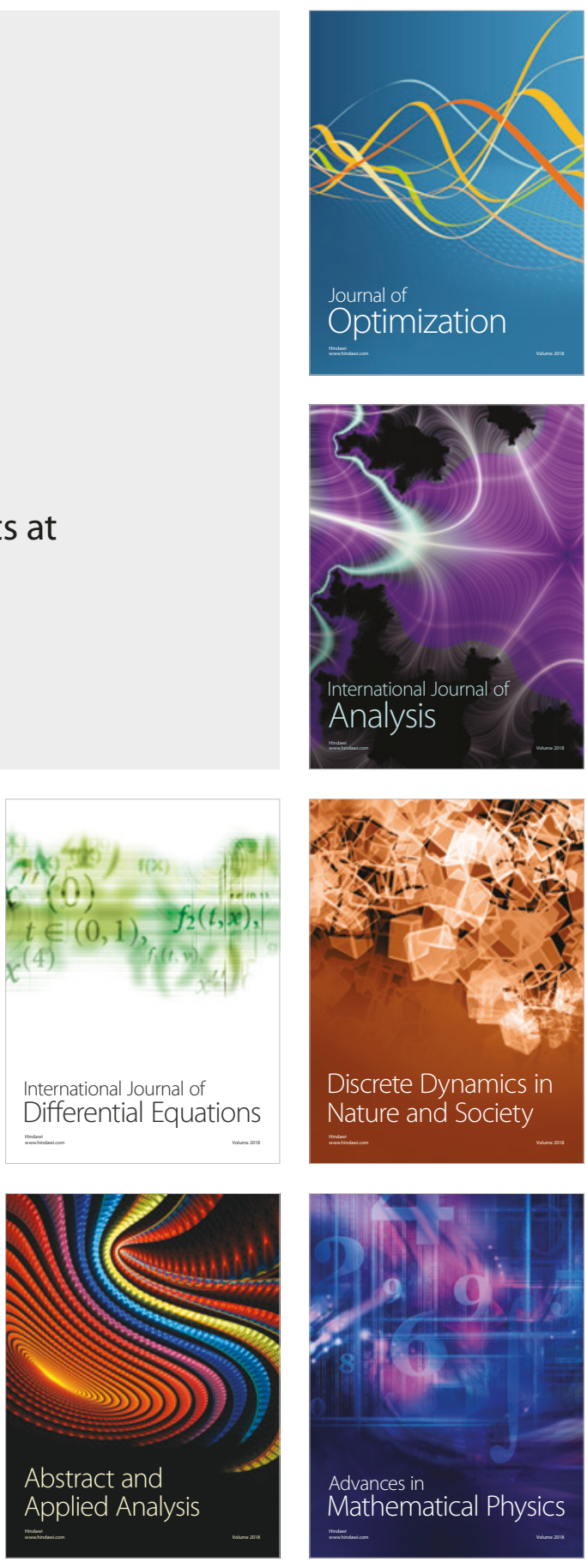